\title{
Universal coverage of renal dialysis in Thailand: promise, progress, and prospects
}

\author{
Sripen Tantivess senior researcher ${ }^{1}$, Pitsaphun Werayingyong researcher ${ }^{1}$, Piyatida Chuengsaman \\ nephrologist $^{2}$, Yot Teerawattananon programme leader ${ }^{1}$
}

${ }^{1}$ Health Intervention and Technology Assessment Program (HITAP), Ministry of Public Health, Nonthaburi, Thailand; ${ }^{2}$ Banphaeo Hospital Public Organization, CAPD Service and Training Centre, Bangkok, Thailand

\begin{abstract}
Thailand's experience in introducing renal replacement therapy as part of its universal health coverage scheme shows the importance of evidence and stakeholders' active participation in all phases of policy development, say Sripen Tantivess and colleagues

Thailand is one of the few developing countries that ensures access to essential health services for all its citizens. Instigated in the early 2000s, the universal health coverage scheme (UCS), extended basic coverage to everyone not already covered by existing public schemes and has been popular, persisting through political instability over the past decade. ${ }^{1}$ The benefits and costs of the UCS have increased since it was introduced. New benefits have included antiretroviral drugs for HIV, in 2004, and renal replacement therapy for end stage renal disease, in 2008. ${ }^{1}$

Renal replacement therapy is expensive and complex, and — unlike HIV/AIDS - kidney diseases afflict a relatively small percentage of the population and have never reached the global or national health agenda. We examine the rationale and factors that influenced the adoption of universal funding of renal replacement therapy, what can be learnt from the decision making process, and the challenges of maintaining funding.
\end{abstract}

\section{Setting the scene}

Health benefit policies in Thailand developed to cover different groups of the population. The civil servant medical benefit scheme for government employees was instigated in 1982 and the social security scheme for formal private employees in 1990. This left a substantial portion of Thai people uninsured. Although healthcare reformers put great efforts into advocating expansion of the health safety net for all, it was not until 2001 that a political party pledged to introduce universal health coverage. The UCS was introduced in 2002, a year after the party's election.

UCS managers adopted almost the same benefit package as offered by the social security scheme because both schemes were funded through capitation. This included chemotherapy and radiation for specific cancers, open heart surgery, prosthetic hip or shoulder replacement, and neurosurgery. ${ }^{2}$ However, because of the relatively high prevalence of end stage renal disease and HIV infection among UCS beneficiaries, the scheme initially excluded treatments for these conditions.

Kidney transplantation has been performed in Thailand since 1972 and has been financed within the civil service scheme since 1980. However, the numbers of donated kidneys have never met the demand ${ }^{3}$ because many people in Thailand believe that their body should remain intact after death, for the next life. ${ }^{4}$ The number of renal transplants in Thai patients has risen from 229 in 2001 to only 308 in 2009, while over 4000 patients wait for a kidney. ${ }^{5}$

Most Thais with end stage renal disease must therefore rely on renal replacement therapy, which is expensive. The two pre-existing public schemes have included peritoneal dialysis and haemodialysis in their benefit packages since 1985 and 1990. However, for those who had to pay for it themselves expenditure on dialysis accounted for $25-70 \%$ of household income. ${ }^{6}$ People coped by reducing the frequency of seeking dialysis; treating anaemia with blood transfusion rather than erythropoietin; restricting spending on transport, food, and education; and borrowing money at high interest rates, something that was especially common in poor households.

\section{Pressure to introduce universal access}

Renal replacement therapy had not originally been covered by the UCS because of its cost. ${ }^{7}$ However, nephrologists and kidney patient groups as well as some health officials and researchers who had helped establish the UCS campaigned for it to be introduced on the grounds of equity, emphasising the disparity between the three public schemes, as well as the catastrophic expenditures incurred by patients on low incomes. Although the patient groups were small and not well known in Thai society, they gained considerable support, not only from longstanding networks of HIV and cancer patients but also from the Thai Nephrologists Association. Furthermore, health policy researchers and nephrologists jointly conducted a series of 
studies to support renal replacement therapy, with their focus on determining appropriate policy options. ${ }^{8}$

In 2004 the National Health Security Office (NHSO), which is responsible for the UCS, commissioned research to determine the value for money of dialysis, including the costs of providing renal replacement therapy in the UCS over 15 years. ${ }^{9}$ It also carried out a survey of public opinion on different options for renal replacement therapy. ${ }^{9}$ Neither peritoneal dialysis nor haemodialysis was shown to be cost effective, but peritoneal dialysis offered better value than haemodialysis. The annual incidence of end stage renal disease was estimated to be $121.9^{10}$ to $158.9^{11}$ per million population ( 7873 to 10016 cases) in 2004 and 2007, respectively. If the government decided to provide universal access to renal replacement therapy, assuming an annual incidence similar to that in developed countries at about 300 per million, ${ }^{12}$ the number of patients receiving dialysis would increase to more than 100000 cases in the tenth year. The NHSO would spend a significant proportion of its annual budget on renal replacement therapy, accounting for 3\% in the first year and $15 \%$ in the fifteenth year.

Although most nephrologists preferred haemodialysis to peritoneal dialysis, all the haemodialysis machines and people with the skills to use them were concentrated in greater Bangkok. This made haemodialysis inaccessible to patients in remote areas. The survey among Thais aged 18-60 years showed that most respondents supported the inclusion of renal replacement therapy in the UCS, and most suggested that if rationing were needed priority should be given to patients with urgent health needs, those who were poor and underprivileged, and bread winners with several child dependants. When asked about a contribution from patients themselves, around $80 \%$ of the respondents were willing to pay 100 baht (£2; €2.5; \$3) a dialysis session, far below the actual cost.

Despite continuing concerns about the cost, mounting evidence suggested that dialysis could be provided more cheaply than originally estimated and with better outcomes. Advocates increased the pressure to fund renal replacement therapy and the government finally agreed to universal funding in October 2007. The decision was influenced by the health minister, ${ }^{13}$ who had long term relationships with health reformists and non-governmental organisations.

\section{Key features of the programme}

The inclusion of renal replacement therapy in the UCS was accompanied by a series of measures to ensure the effectiveness of the coverage and efficient use of resources. The first element was to strengthen measures to prevent end stage renal disease by encouraging the early detection and treatment of hypertension and diabetes through community screening, with financial incentives for health workers.

Secondly, a policy of using peritoneal dialysis first was introduced, with haemodialysis as a second line treatment for those not suitable for peritoneal dialysis. Although nephrologists initially opposed this because of their poor experience with peritoneal dialysis, they accepted it because it was the only way that poorer patients would be able to obtain dialysis. ${ }^{9}$ In addition, peritoneal dialysis could be administered on a "self care" basis in patients' homes, saving them travel costs for hospital haemodialysis.

The third element involved financing. While most ambulatory services in the UCS are paid for through capitation payments, the peritoneal dialysis first policy was incentivised through a fixed fee for each patient started and maintained on peritoneal dialysis. ${ }^{14}$ Patients who seek haemodialysis as first line treatment have to shoulder the costs. Cost containment measures-namely central tendering and bulk purchasing-were also introduced for medicines and supplies.

The NHSO encouraged the establishment of peritoneal dialysis in district hospitals and other public healthcare facilities. An advantage of operating peritoneal dialysis centres in district hospitals is that these hospitals are well connected with comprehensive primary care networks at the subdistrict and community levels. It also created treatment partnerships with private facilities to overcome the limited capacity in the government sector for both haemodialysis and peritoneal dialysis, setting fixed prices for reimbursement.

In response to the prevailing shortages of physicians and nurses, the NHSO and its partners organised training in peritoneal dialysis and related care for these and other health professionals, such as nutritionists. ${ }^{15}$ Task shifting was another crucial strategy. As peritoneal dialysis centres reach patients in communities through existing primary care networks, subdistrict health workers and village volunteers, patient groups, and even individual patients and family members were also trained to provide information and education.

Finally, a renal disease registry was set up to provide information on resources and patient profiles for strategic management, planning, quality assurance, and regulation. The NHSO also set up an inventory and procurement system connecting the providers of peritoneal dialysis with suppliers of medicines and materials; this is used for inventory control and to ensure timely delivery of erythropoietin, dialysates for peritoneal dialysis, and catheters.

\section{Effect of the new policy}

The universal renal replacement therapy programmes have been continually developed since 2008. Between January 2008 and 2012 the number of peritoneal dialysis units increased from 23 to 160 and the number of peritoneal dialysis nurses from 56 to 423; 345 physicians were trained in inserting Tenckhoff catheters. ${ }^{15}$

UCS patients who had paid for haemodialysis before October 2008 and decided to continue haemodialysis were required to pay 500 baht per session, while the NHSO subsidised the remaining cost (1000-1200 baht). There is no subsidy for patients who start peritoneal dialysis but choose to switch to haemodialysis in the absence of contraindications. The reimbursement of erythropoietin started in 2009. Initially it was funded only for patients complying with the peritoneal dialysis first policy but was provided to all dialysis patients registered in the NHSO database from $2011 .^{15}$

The number of patients having peritoneal dialysis increased steeply after 2008, even though the number of peritoneal dialysis units, which are mostly in public health facilities $(90 \%)$, reached a plateau, indicating that peritoneal dialysis units were able to increase their capacity (fig $1 \Downarrow$ ). Haemodialysis units face more difficulty in meeting larger demands because they rely heavily on trained nurses and machines.

There are no data on the numbers of patients who paid for renal replacement therapy before 2008 . The analysis of life expectancy of dialysis patients before 2008 was based on registered patients under the civil service and social security schemes. ${ }^{9}$ There are no data to compare life expectancy of those diagnosed with end stage renal dialysis before and after the introduction of the universal dialysis policy, but we can assume that before the programme $90 \%$ of patients died within 3-6 months. At present patients may survive for at least 5-10 years. ${ }^{16}$ 
Analysis of catastrophic spending on health problems-defined as household health expenditures $\geq 10 \%$ of total expenditure-show that the introduction of the UCS in 2001 benefited the poor more than the rich (fig $2 \Downarrow$ ). ${ }^{17}$ The expansion of universal access to antiretroviral treatment during 2002-07 resulted in another decline in the incidence of catastrophic health expenditure in both rich and poor households. ${ }^{14}$ However, there is insufficient evidence that the introduction of universal renal replacement therapy has had a further effect on catastrophic health expenditure, though it may be too early to tell.

\section{Lessons for decision makers}

Thailand's success in introducing a universal health coverage that includes high cost interventions such as renal replacement therapy provides valuable lessons for other settings.

Firstly, evidence is necessary for policy development, particularly in decisions about covering high cost interventions in resource limited settings. Local evidence played a crucial role in the adoption and implementation of universal renal replacement therapy in Thailand. This also reflects the need for local capacity in policy and health systems research.

Secondly, the participation of key stakeholders, including politicians, health providers, professional associations, academics, and researchers, is vital. This is not only to increase the sense of ownership of such a policy but also to reduce conflicts between different interests. Information sharing among stakeholders was successful in making health professionals, who had favoured haemodialysis, accept the peritoneal dialysis-first policy.

Since resources are scarce, it is important to emphasise to everyone that rational allocation of health resources is best practice. The philosophy behind universal health coverage means that everybody in society recognises the limitations of what the government can offer and tries to find the best solution to particular problems. Not everybody can get what they think is the best treatment, but everybody can get good treatment.

Finally, although agencies such as the World Health Organization, underline "health financing" as a key element of universal health coverage, ${ }^{18}$ Thailand's renal replacement therapy policy shows that health financing is not the sole factor for achieving the policy goal. Strengthening the capacity of the health system, including workforce development, selection of appropriate health technologies, and effective monitoring and evaluation are also important.

\section{Challenges and opportunities}

Despite the careful implementation of funding for renal replacement therapy, the sustainability of this ambitious policy is in question, given the rising incidence of end stage renal disease and the proportion of the UCS budget devoted to dialysis (table $\Downarrow$ ). This may reflect inadequate control of hypertension, despite the efforts to control it. Though the number of people needing dialysis has escalated, renal transplant services still face a shortage of kidney donors. ${ }^{19}$

Some nephrologists are pressing the NHSO to revoke its peritoneal dialysis first policy because they believe that haemodialysis is better and that the NHSO is offering a second class treatment. This campaign is in line with the pressure being exerted by private providers, who support an extension of haemodialysis. Research has suggested that after all possible confounders were adjusted for, patients who start haemodialysis as first line treatment under the other insurance schemes live longer than those treated under the peritoneal dialysis first policy (odds ratio $=3.25) .{ }^{16}$ But different mortality rates were observed across regions, with those having dialysis by either means in greater Bangkok having a better chance of survival than those in the north east and south of the country. If UCS patients were offered haemodialysis from the outset the NHSO would need to invest annually in an additional 1000 haemodialysis machines and 500 trained haemodialysis nurses, which are unlikely to be affordable in the long run. Also, evidence from the US and Canada suggests that although the mortality rate of people having peritoneal dialysis was relatively higher than for those being treated with haemodialysis during the first few years after peritoneal dialysis was introduced, the gap was eliminated once nephrologists became more competent in peritoneal dialysis. ${ }^{20} 21$ Therefore the NHSO's renal replacement therapy programmes need to put more effort into improving the quality of peritoneal dialysis through professional training.

The Thai policy on renal replacement therapy remains an unfinished agenda and continues to be central to policy debates. In this it reflects debates in many health systems about high cost treatments and how to balance equity, cost effectiveness, and affordability.

We thank Suwit Wibulpolprasert, Prateep Dhanakijcharoen, Surapol Aruyapitipan, and Jadej Thammatacharee for their helpful comments. The Health Intervention and Technology Assessment Program (HITAP) is funded by the Thailand Research Fund through a senior research scholar on health technology assessment award (RTA5580010), the National Health Security Office, the Thai Health Promotion Foundation, the Health System Research Institute, and the Bureau of Health Policy and Strategy, Ministry of Public Health.

Contributors and sources: All authors contributed to the idea and content of the manuscript. YT is the guarantor.

Competing interests: YT was a principal investigator of the economic evaluation on policy options for end stage renal disease that was subsequently used to inform the policy on renal dialysis. PC is a nephrologist and consultant to the National Health Security Office.

Provenance and peer review: Commissioned; externally peer reviewed.

1 Evans TG, Chowdhury AMR, Evans DB, Fidler AH, Lindelow M, Mills A, et al. Thailand's Universal Coverage Scheme: Achievements and Challenges. An independent assessment of the first 10 years (2001-2010). Health Insurance System Research Office, 2012.

2 Teerawattananon $Y$, Tangcharoensathien $\mathrm{V}$. Designing a reproductive health services package in the universal health insurance scheme in Thailand: match and mismatch of need, demand and supply. Health Policy Plan 2004;19(suppl 1):i31-9.

3 Tungsiripat $\mathrm{R}$, Tangcharoensathien $\mathrm{V}$. Regulation of organ transplantation in Thailand: does it work? HEFP working paper 04/03. London School Of Hygiene and Tropical Medicine, 2003.

4 Anothayanon $\mathrm{K}$, Luanratanakorn $\mathrm{P}$, Teerawattananon $\mathrm{Y}$. Attitude and willingness towards organ donation: the survey for policy recommendations. J Health Sci 2002;11:826-35.

5 Praditpornsilpa K, Lekhyananda S, Premasathian N, Kingwatanakul P, Lumpaopong A, Gojaseni $\mathrm{P}$, et al. Prevalence trend of renal replacement therapy in Thailand: impact of health economics policy. J Med Assoc Thai 2011;94(suppl 4):S1-6.

6 Prakongsai P, Palmer N, Uay-Trakul P, Tangcharoensathien V, Mills A. The implications of benefit package design: the impact on poor Thai households of excluding renal replacement therapy. J Int Dev 2009;21:291-308.

7 Treerutkuarkul A. Thailand: health care for all, at a price. Bull World Health Organ 2010;88:84-5.

8 Prakongsai P, Tangcharoensathien V, Kasemsup V, Teerawattananon Y, Supaporn T, Vasavid C. Policy recommendations on extending access to renal replacement therapy under universal coverage in Thailand. J Health Sci 2006;15:617-31.

9 Teerawattananon $\mathrm{Y}$, Mugford M, Tangcharoensathien V. Economic evaluation of palliative management versus peritoneal dialysis and hemodialysis for end-stage renal disease: evidence for coverage decisions in Thailand. Value Health 2007;10:61-72.

10 Thai Renal Replacement Therayp (TRT) Committee. TRT registry annual report. Nephrology Seciety of Thailand, 2005.

11 Thai Renal Replacement Therayp (TRT) Committee. Provision of RRT in Thailand in year 2007. In: Praditpornsilp K, ed. Thailand renal replacement therapy: year 2007. Nephrology Seciety of Thailand, 2008.

12 United States Renal Data System. Annual data report 2001: incidence and prevalence . USRDS Coordinating Center, 2004.

13 Patcharanarumol W, Tangcharoensathien V, Limwattananon S, Panichkriengkrai W, Pachanee K, Poungkantha W, et al. Why and how did Thailand achieve good health at low cost? In: Balabanova D, McKee M, Mills A, eds. Good health at low cost: 25 years on What makes a successful health system? London School of Hygiene and Tropical Medicine, 2011.

14 National Health Security Office. Fund management manual of national health security [in Thai]. National Health Security Office, 2007. 
15 Dhanakijcharoen P, Sirivongs D, Aruyapitipan S, Chuengsaman P, Lumpaopong A. The "PD First" policy in Thailand: three-years experiences (2008-2011). J Med Assoc Thai 2011;94(suppl. 4):S153-61.

16 Survival analysis of end-stage renal disease patients in Thailand. Stakeholder meeting; 2012 September 28th Department of Health, Ministry of Public Health.

17 Limwattananon S, Tangcharoensathien V, Prakongsai P. Catastrophic and poverty impacts of health payments: results from national household surveys in Thailand. Bull World Health Organ 2007:85:600-6

18 World Health Organization. The world health report: health system financing: the path to universal coverage . Geneva. Switzerland: World Health Organization, 2010.

19 Kearkiat P. Report of the Thailand renal replacement therapy year 2010. Nephrology Society of Thailand, 2012.
20 Mehrotra R, Chiu YW, Kalantar-Zadeh K, Bargman J, Vonesh E. Similar outcomes with hemodialysis and peritoneal dialysis in patients with end-stage renal disease. Arch Intern Med 2011;171:110-8.

21 Yeates K, Zhu N, Vonesh E, Trpeski L, Blake P, Fenton S. Hemodialysis and peritoneal dialysis are associated with similar outcomes for end-stage renal disease treatment in Canada. Nephrol Dial Transplant 2012;27:3568-75.

Accepted: 13 January 2013

Cite this as: BMJ 2013;346:1462

(c) BMJ Publishing Group Ltd 2013 


\section{Table}

Table 1| Budget allocation to renal replacement therapy compared with total budget (million baht), 2008-12

Fiscal year Total budget Renal replacement therapy (\%)

\begin{tabular}{lll}
2008 & 76800 & $160(0.2)$ \\
\hline 2009 & 80600 & $1400(1.7)$ \\
\hline 2010 & 89400 & $2700(3.0)$ \\
\hline 2011 & 101100 & $3200(3.2)$ \\
\hline 2012 & 114500 & $3900(3.4)$ \\
\hline
\end{tabular}

100 baht $=£ 2 ; € 2.5 ; \$ 3$. 


\section{Figures}

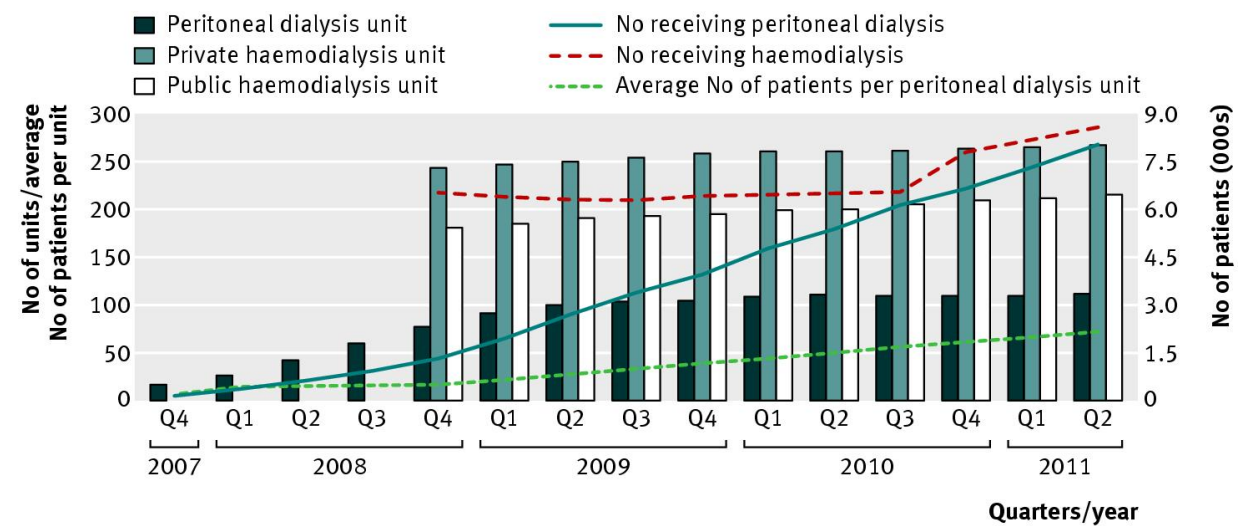

Fig 1 Numbers of peritoneal dialysis and haemodialysis units, patients having each type of dialysis, and the average number of patients per peritoneal dialysis unit

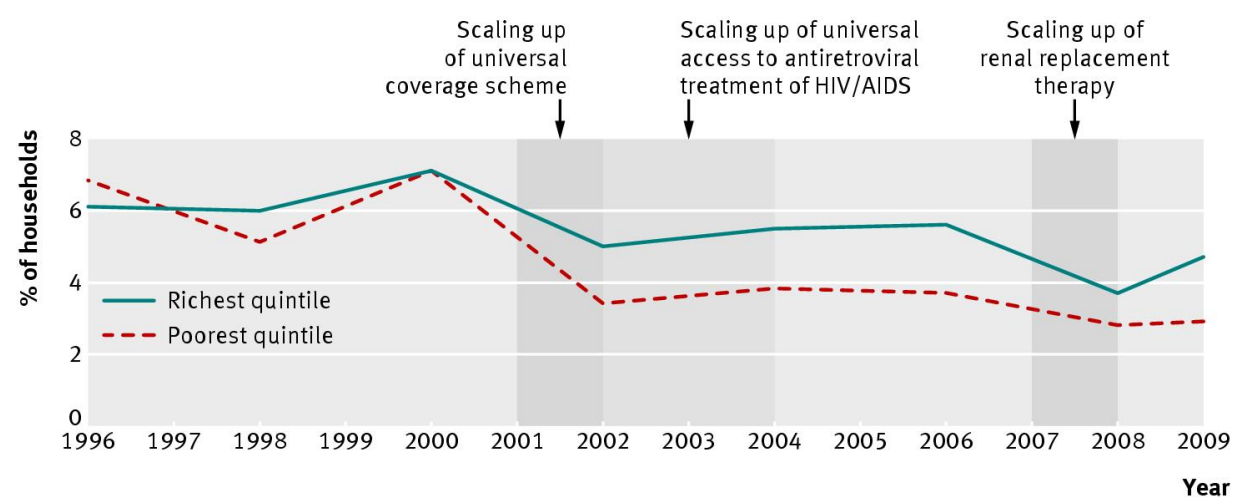

Fig 2 Proportion of households in which health accounts for more than $10 \%$ of total expenditure among richest and poorest quintiles 